\title{
La longevidad del besugo Epigonus crassicaudus en Chile: ¿es similar a la de sus parientes?
}

\author{
Longevity of cardinalfish Epigonus crassicaudus in Chile: is it similar to that of its relatives? \\ Vilma Ojeda ${ }^{1}$, Rodrigo Wiff ${ }^{1}$, Carla Labrín ${ }^{1}$ y Francisco Contreras ${ }^{1}$ \\ ${ }^{1}$ División de Investigación Pesquera, Instituto de Fomento Pesquero, Blanco No 839, Valparaíso, Chile. vojeda@ifop.cl

\begin{abstract}
The cardinalfish Epigonus crassicaudus is a deep-demersal fish with commercial importance for the trawl fishery operating off central Chile. Previous analysis in age assignment, based on the whole otolith, determined the maximum age to be 15 years old. Although scarce data is available for the genus, other congeneric species might live up to 100 years. Using sagitta otolith transversal sections, we determined age to 1277 fish collected in central Chile between J anuary and December 2008. Longevity was determined in 54 years old.
\end{abstract}

Key words: Deep-water fisheries, lifespan, age-assignment

\section{INTRODUCCIÓN}

El besugo Epigonus crassicaudus de Buen, 1959 es un pez caracterizado por hábitos mesobento/pelágicos cuyos individuos adultos habitan el talud continental y montes submarinos donde forman densas agregaciones, mientras que los juveniles se encuentran desagregados en la zona pelágica cercana a la plataforma continental (Parin 1987). El besugo es una especie endémica de las costas frente a Chile y de acuerdo con la información pesquera, las mayores abundancias se encuentra distribuidas entre los $29^{\circ} 00^{\prime} \mathrm{S}$ y $42^{\circ} 50^{\prime} \mathrm{S}$ (Leal et al. 2009) entre los 200 y 400 $\mathrm{m}$ de profundidad (Wiff et al. 2008).

Estudios de edad y crecimiento, basados en la asignación de edades a otolitos enteros, señalaban que Epigonus crassicaudus tiene una longevidad de 15 años (Gálvez et al. 2000), lo cual recientemente fue confirmado por Cubillos et al. (2009a). Estudios preliminares indican que especies congenéricas tienen una longevidad mediana entre 7 y 15 años (Abramov 1990). Sin embargo otros estudios mas acabados indican que otras especies congenéricas se caracterizan por su extremadamente baja tasa de crecimiento, gran longevidad y baja tasa de mortalidad natural. En este contexto, Tracey et al. (2000) y Neil et al. (2008) usando técnicas de secciones transversales de otolitos validadas con radiometría y bomba de radiocarbono, señalan que Epigonus telescopus en Nueva Zelanda podría tener una longevidad cercana a los 100 años de edad. Así, E. crassicaudus presentaría características de historias de vida totalmente disímil a su pariente mejor estudiado, E. telescopus. Estudios de longevidad son de importancia para la sustentabilidad de los recursos, toda vez que errores de asignación de esta puede producir profundas diferencias en la explotación pesquera. Desde este punto de vista, en el presente trabajo se reporta el resultado del análisis de otolitos enteros y secciones transversales para besugo en Chile con el objetivo de verificar si la longevidad del recurso corresponde o no a la de un pez 'típico' de aguas profundas.

\section{Material y Métodos}

El análisis es basado en muestras aleatorias de otolitos sagitales provenientes desde el área comprendida entre $33^{\circ} 48^{\prime} 18^{\prime \prime}-39^{\circ} 49^{\prime} 30^{\prime \prime}$ ' , colectadas entre enero y diciembre de 2008 por buques arrastreros industriales que normalmente operan en la pesquería demersal centro-sur de Chile. Para la lectura de anillos de crecimiento se emplearon tanto los otolitos enteros (observación en superficie, por su cara externa) como también en láminas finas de sección transversal a través del foco, lo que equivale a estudiarlos hacia su cara interna. Se dispuso el otolito izquierdo para el análisis de los otolitos enteros y el otolito derecho para ser empleado en la obtención de las secciones transversales. El tratamiento previo a la asignación de edad del otolito entero consistió en un lavado con agua y pincel y secado del otolito en el horno por $8 \mathrm{~h}$ a una temperatura de $60^{\circ} \mathrm{C}$ para efectos de homogenización de la muestra previo al pesaje. Se le asignaron edades a un total de 1277 otolitos provenientes de ejemplares entre 18 y $40 \mathrm{~cm}$ de longitud total (LT, medidos al cm más cercano), los cuales correspondieron a 536 machos, 721 hembras y 20 de sexo indeterminado. 
La determinación de edad del pez de acuerdo a la lectura del otolito entero se llevó a cabo observando las estructuras bajo microscopio estereoscópico con aumento de 10x en general y aumentos mayores para detalles hacia la periferia. En el caso de la sección trasversal y previo a la obtención de la lámina, el otolito se hornea a $250^{\circ} \mathrm{C}$ por un período entre 10 y 15 min hasta obtener un tono tostado y posteriormente proceder a la inclusión en bloques de resina lo que facilita el seccionamiento a nivel de foco. Se extraen láminas de 0,5 mm con máquinas de corte de precisión. Al momento de observación de la muestra se cubre con aceite transparente de uso común en microscopía y se observa con magnificación 20x (Para mayor detalle ver Ojeda \& Muñoz 2008).

\section{Resultados y Discusión}

Los otolitos de besugo son de forma poligonal, cuya periferia presenta crenulaciones irregulares (Fig. 1). En los otolitos enteros es posible medir con relativa facilidad los radios de los anillos de crecimiento especialmente cuando tienen menos de 7 annuli (Fig. 1a). Sin embargo, la lectura de otolitos con más de 7 annuli resulta dificultosa y se torna incierta la clasificación del material en formación, especialmente en la zona cercana al borde, debido a que los anillos se encuentran poco espaciados, haciendo impracticable distinguir, discriminar y contabilizar anillos de crecimiento en tal ángulo del crecimiento. Esta disminución en la tasa de crecimiento posiblemente sea producto de una canalización de la energía a reproducción (Kozlowski \& Teriokhin 1999). Por otra parte, cuando se observan las secciones transversales de los otolitos (Fig. 1b, c) se presenta una secuencia de anillos más fácil de contabilizar desde el foco del otolito hasta la periferia, caracterizando esta técnica como más adecuada para la asignación de edad en comparación con el uso del otolito entero.

La Tabla 1 presenta una comparación de la lectura de los anillos bajo las dos técnicas mencionadas (otolitos enteros y láminas transversales, cada técnica aplicada en un otolito del par). Esta tabla de simetría consta de entradas constituidas por las edades determinadas con estas dos técnicas y muestra la diferencia en edades de una misma

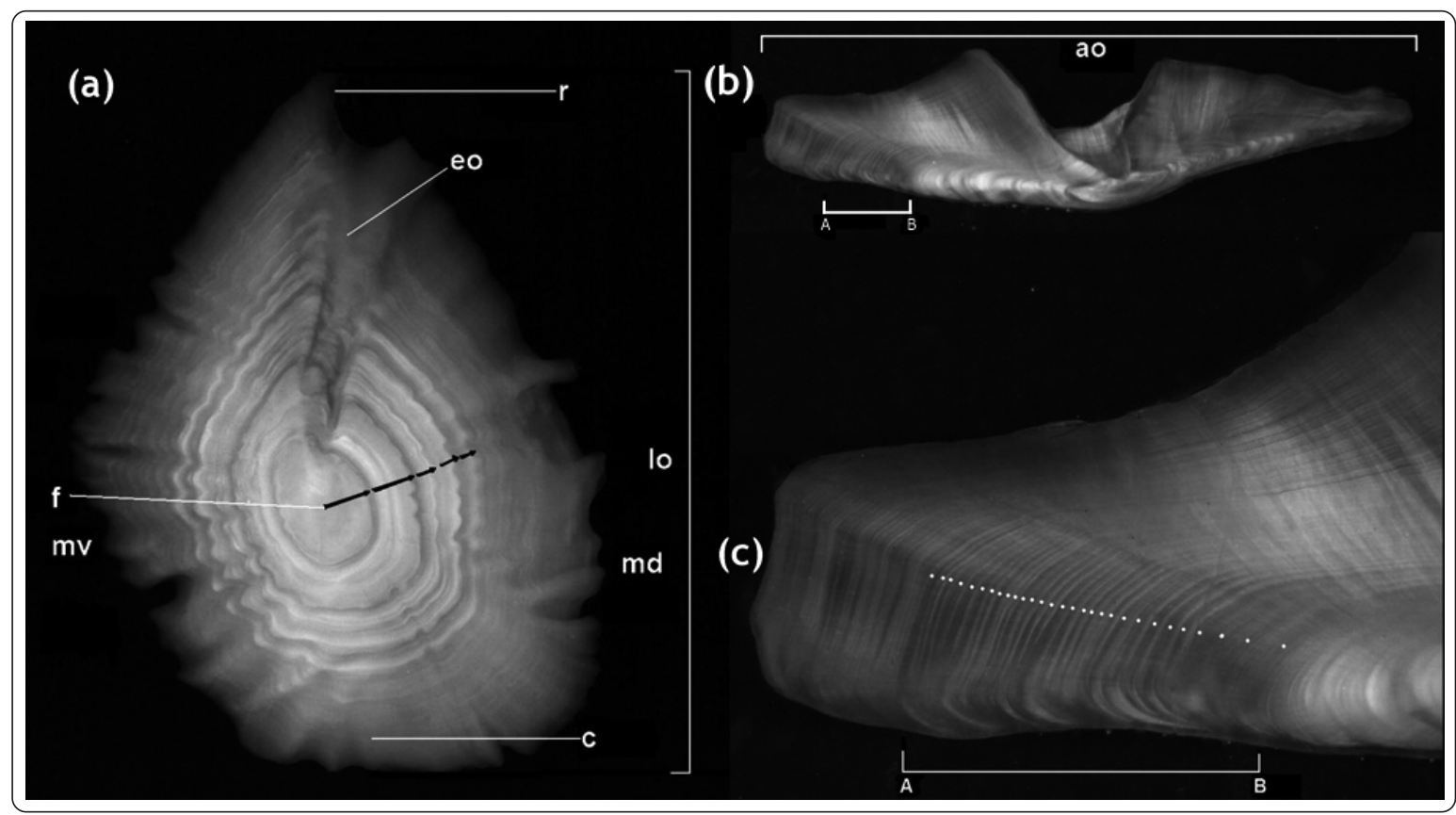

Figura 1. Fotografías de otolitos de besugo (10x). (a) Otolito entero. Las flechas negras señalan los primeros anillos de crecimiento. (b) Lámina transversal obtenida a través del foco (20x). (c) Lámina transversal ampliada para señalar algunos anillos de crecimiento con puntos blancos Trazo AB como referencia de la zona ampliada de la fotografía b; $r=z o n a$ rostral; $c=$ zona caudal; $m v=$ margen ventral; $m d=$ margen dorsal; $f=f o c o ; e 0=$ excisura ostii; lo= longitud del otolito; ao= alto otolito / Photographs of cardinalfish's otoliths (10x). (a) The whole otolith. Black arrows indicate the first few rings. (b) Transversal section obtained across the focus (20x) (c) Transversal section magnified to point out yearly growth rings in white dots. Line $A B$

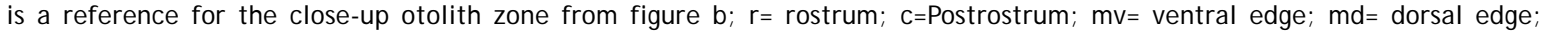
$\mathrm{f}=$ focus; $\mathrm{e}=$ =xcisura ostii; $10=$ otolith maximum length; $\mathrm{a}=$ = otolith height 
muestra cuando los otolitos enteros o láminas transversales fueron observados. Nótese que a medida que se está en presencia de edades más adultas, mayor es la diferencia entre las técnicas de asignación de edades. Lo expresado anteriormente es una situación común que se presenta en peces de longevidad media ó alta (Chilton \& Beamish 1982, Ojeda \& Aguayo 1986, CARE 2006), razón por la cual es esencial determinar la edad en el ángulo de observación que presente un mejor despliegue de información que asegure la adecuada visualización de los annuli en todo el campo de la estructura. En este contexto, se consideraron las edades obtenidas en base a la observación de las secciones transversales de los otolitos para construir una matriz edad-longitud corporal, la cual está formada por individuos entre 6 y 54 años de edad (Tabla 2).

Cubillos et al. (2009b) no encuentran grandes diferencias entre la lectura de los annuli leídos en otolitos enteros y secciones transversales de E. crassicaudus. La incapacidad de observación de edades longevas en secciones transversales de otolitos en Cubillos et al. (2009b) podría ser causada por: (1) el bajo número de muestras utilizadas por estos autores, donde sólo a 53 otolitos se les asignó edades a secciones transversales, (2) se utilizaron secciones transversales sin tostado, lo cual dificulta la lectura de anillos más longevos, (3) los ejemplos presentados por los autores en secciones transversales muestran individuos jóvenes, en los cuales los anillos de crecimiento son bastante visibles, ya sea en el otolito entero o en su sección transversal. El otolito con la edad aumenta en espesor, aumentando el número de anillos a leer hacia la cara interna de la lámina extraída, lo que puede producir errores en la lectura.

Por otra parte, Cubillos et al. (2009b) realizaron una validación de los anillos de crecimiento observados tanto para el primer annuli a través de recuento de microincrementos como para edades mayores utilizando una técnica basada en mediciones de decaimiento de radioisótopos. Para los peces de edades mayores, estos autores concluyeron que su uso confirmaba las edades en promedio observadas en los otolitos enteros. Aunque esta técnica es adecuada para la determinación de edad en especies longevas (Campana 2001), en el contexto que Cubillos et al. (2009b) la utilizan, puede llevar a subestimaciones severas de la longevidad dado que emplearon muestras agrupadas por talla de intervalos de 2 a $3 \mathrm{~cm}$, sin embargo, la agrupación de otolitos por tallas puede causar una invalidación de la técnica de radiometría en la asignación de edades (Andrews \& Tracey 2007).

Por otro lado, la agrupación de individuos de igual longitud podría contener varios grupos de edad, debido a que la edad no siempre esta bien correlacionada con la longitud corporal de peces (Prajneshu \& Venugopalan 1999). Este problema puede ser particularmente importante de tratar en especies longevas donde la variación de la longitud a la edad es alta (Mitton \& Lewis 1989) y donde esta variante se incrementa hacia edades mayores por un proceso conocido como depensación del crecimiento (Pfister \& Stevens 2002).

Tabla 1. Comparación de las asignaciones de edades en besugo empleando otolitos enteros y láminas finas transversales / Comparison of age assignation in cardinalfish using whole otoliths and traversal sections

\begin{tabular}{|c|c|c|c|c|c|c|c|c|c|c|c|c|c|c|c|c|c|c|c|c|c|c|c|c|c|c|c|c|c|c|c|}
\hline \multirow{2}{*}{\multicolumn{2}{|c|}{ EDAD }} & \multicolumn{30}{|c|}{$\mathrm{N}^{\circ}$ Anillos observados en láminas de otolitos, seccionados transversalmente } \\
\hline & & 7 & 8 & 9 & 10 & 11 & 12 & 13 & 14 & 15 & 16 & 17 & 18 & 19 & 20 & 21 & 22 & 23 & 24 & 25 & 26 & 27 & 28 & 29 & 30 & 31 & 32 & 33 & 34 & 35 & Total \\
\hline \multirow{5}{*}{ 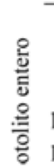 } & 7 & 1 & & 1 & & & & & & 1 & & & & & & & & & & & & & & & & & & & & & 3 \\
\hline & 8 & & & 3 & & 1 & & & & & & & & & & & & & & & & & & & & & & & & & 4 \\
\hline & 9 & & 2 & 2 & 1 & 3 & & & 1 & & & & & & & & & & & & & & & & & & & & & & 9 \\
\hline & 10 & & 1 & 2 & 1 & 1 & 1 & & & & & 1 & & & & & & & & & & & & & & & & & & & 7 \\
\hline & 11 & & & 1 & 1 & & 1 & & & & & & & & & & & & & & & & & & & & & & & & 3 \\
\hline \multirow{4}{*}{ 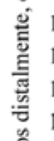 } & 12 & & & & 1 & & 1 & & 1 & 2 & & & 1 & & & & & & & & & & & & & & & & & & 6 \\
\hline & 13 & & & & & & & & & & & & & & & & 1 & & & & & & & & & & & & & & 1 \\
\hline & 14 & & & & & & 2 & 1 & & 1 & & & & & & 1 & 1 & & 1 & & & & & & & & & & & & 7 \\
\hline & 15 & & & & & & & 2 & & & & & 1 & & & & & & & & & & & & & & & & & & 3 \\
\hline \multirow{6}{*}{ 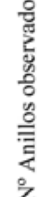 } & 16 & & & & & & & & 1 & & 1 & 1 & 2 & & 1 & 2 & & & & 1 & & & & 1 & & & & & & & 10 \\
\hline & 17 & & & & & & & & & & & & 1 & & & 1 & 1 & & & & & & 1 & & & & & & & & 4 \\
\hline & 18 & & & & & & & & & & & 1 & & & & 1 & & 3 & 1 & & & & & & & & 1 & & & & 7 \\
\hline & 19 & & & & & & & & & & 1 & & 1 & & & & 2 & & 1 & & & & & & & & 2 & & & & 7 \\
\hline & 20 & & & & & & & & & & & & 1 & 1 & & & & 1 & 2 & 1 & & & 1 & & & & 1 & & & 1 & 9 \\
\hline & 21 & & & & & & & & & & & & & & 1 & & 1 & 2 & 1 & & & 1 & & & & & 3 & 1 & & & 10 \\
\hline & 22 & & & & & & & & & & & & & & & & & & & & & & & & & & & & & & 0 \\
\hline \multicolumn{2}{|c|}{ Total } & 1 & 3 & 9 & 4 & 5 & 5 & 3 & 3 & 4 & 2 & 3 & 7 & 1 & 2 & 5 & 6 & 6 & 6 & 2 & 0 & 1 & 2 & 1 & 0 & 0 & 7 & 1 & 0 & 1 & 90 \\
\hline
\end{tabular}


A su vez, Cubillos et al. (2009b) informan una función exponencial para dar cuenta de la relación existente entre la masa del otolito y la longitud de los individuos, lo que podría limitar severamente la asignación de edades, debido a que en tallas mayores, para un rango pequeño de longitudes, continúa el aumento en masa de otolitos, con la consiguiente incorporación de radioisótopos.

Una forma de solucionar este problema es usando la técnica de radiometría sobre núcleos de otolitos y luego de agrupar edades conocidas para la validación final de la edad (Andrews \& Tracey 2007). Si bien es cierto que en el presente estudio no se valida la asignación de edades, pone en evidencia que podríamos estar observando muchas más clases anuales que las que realmente se han reportado para esta especie. En la literatura se encuentran variados ejemplos de como errores en la asignación de edades han contribuido a generar una seria sobreexplotación de los recursos (Campana 2001). El problema común es una subestimación de la longevidad (más que una sobreestimación), generando al final tasas de crecimiento y características de resiliencia más optimistas.

Conocemos que la mortalidad natural $(M)$ es inversamente dependiente de la longevidad $\left(T_{\max }\right)$ cuya forma funcional es $M=3 / T_{\max }$ (Hewitt \& Hoenig 2005), $M$ sería igual a $0,2\left(\right.$ año $\left.{ }^{-1}\right)$ y $0,06\left(\right.$ año $\left.{ }^{-1}\right)$ para el caso de longevidad 15 y 54, respectivamente. Esto llevaría a un escenario de explotación totalmente diferente. Tomando la aproximación simplista de Gulland (1971), conocemos que la razón entre una captura sustentable $(Y)$ y la biomasa virginal $(B o)$ esta descrita por $Y$ / Bo = 0,5 M. Así la razón de explotación en caso de longevidad igual a 15 años seria 0,1 año $0^{-1}$ y 0,03 año ${ }^{-1}$ en el caso de ser 54 años la longevidad del recurso. Esto significa que a un nivel de Bo conocido, una captura sustentable recomendada bajo la longevidad de 15 años sería muchísimo mayor (más de 300\%) que si la longevidad incorporada al cálculo fuese 54 años.

\section{Agradecimientos}

Se expresa agradecimiento a los integrantes del proyecto Seguimiento de las Pesquerías Demersales Centro Sur y

Tabla 2. Clave edad-talla para las asignaciones de edades desde la sección transversal en otolitos de besugo / Age-atlength key for the age assignation by rings counting from the transversal section of otolith in cardinalfish

\begin{tabular}{|c|c|c|c|c|c|c|c|c|c|c|c|c|c|c|c|c|c|c|c|c|c|c|c|c|c|c|}
\hline \multirow{2}{*}{$\begin{array}{l}\text { Tallas } \\
(\mathrm{cm})\end{array}$} & \multirow[t]{2}{*}{ Total } & \multicolumn{25}{|c|}{ GRUPOS DE EDAD } \\
\hline & & 6 & 8 & 10 & 12 & 14 & 16 & 18 & 20 & 22 & 24 & 26 & 28 & 30 & 32 & 34 & 36 & 38 & 40 & 42 & 44 & 46 & 48 & 50 & 52 & 54 \\
\hline \multicolumn{27}{|l|}{15} \\
\hline \multicolumn{27}{|l|}{16} \\
\hline \multicolumn{27}{|l|}{17} \\
\hline 18 & 1 & 1 & & & & & & & & & & & & & & & & & & & & & & & & \\
\hline 19 & 1 & 1 & & & & & & & & & & & & & & & & & & & & & & & & \\
\hline 20 & 7 & 3 & 1 & 2 & 1 & & & & & & & & & & & & & & & & & & & & & \\
\hline 21 & 15 & 2 & 2 & 6 & 5 & & & & & & & & & & & & & & & & & & & & & \\
\hline 22 & 29 & 2 & 7 & 11 & 5 & 4 & & & & & & & & & & & & & & & & & & & & \\
\hline 23 & 17 & 4 & 5 & 5 & & 2 & 1 & & & & & & & & & & & & & & & & & & & \\
\hline 24 & 38 & & 3 & 15 & 12 & 5 & 3 & & & & & & & & & & & & & & & & & & & \\
\hline 25 & 40 & & 14 & 5 & 8 & 11 & 1 & 1 & & & & & & & & & & & & & & & & & & \\
\hline 26 & 43 & 3 & 8 & 13 & 9 & 6 & 3 & 1 & & & & & & & & & & & & & & & & & & \\
\hline 27 & 41 & & 1 & 6 & 8 & 9 & 8 & 5 & 2 & 1 & 1 & & & & & & & & & & & & & & & \\
\hline 28 & 39 & & 1 & 1 & 4 & 12 & 10 & 5 & 3 & 2 & 1 & & & & & & & & & & & & & & & \\
\hline 29 & 44 & & & 2 & 2 & 6 & 6 & 5 & 9 & 5 & 6 & 2 & 1 & & & & & & & & & & & & & \\
\hline 30 & 43 & & & 1 & & 6 & 5 & 4 & 4 & 7 & 5 & 3 & 5 & 3 & & & & & & & & & & & & \\
\hline 31 & 92 & & & & 2 & & 1 & 7 & 7 & 11 & 13 & 13 & 13 & 9 & 6 & 4 & 2 & 2 & 1 & 1 & & & & & & \\
\hline 32 & 147 & & & & 1 & 1 & 1 & 2 & 7 & 13 & 12 & 12 & 19 & 16 & 17 & 15 & 9 & 6 & 6 & 7 & & 1 & & 1 & 1 & \\
\hline 33 & 212 & & & & & 2 & 2 & 2 & 9 & 13 & 14 & 23 & 22 & 25 & 32 & 14 & 13 & 16 & 8 & 6 & 3 & 3 & 2 & 1 & 2 & \\
\hline 34 & 216 & & & & & & 1 & 1 & 4 & 4 & 12 & 30 & 35 & 25 & 17 & 21 & 20 & 14 & 12 & 7 & 6 & 3 & 1 & 3 & & \\
\hline 35 & 137 & & & & & & & 1 & 3 & 5 & 12 & 15 & 14 & 15 & 16 & 15 & 10 & 10 & 10 & 6 & 2 & & 1 & 1 & & 1 \\
\hline 36 & 72 & & & & & & & & 1 & & 4 & 6 & 10 & 10 & 13 & 8 & 6 & 3 & 4 & 3 & 2 & 2 & & & & \\
\hline 37 & 31 & & & & & & & & & & 2 & 2 & 4 & 7 & 1 & 4 & 3 & 1 & 4 & & 3 & & & & & \\
\hline 38 & 6 & & & & & & & & & & & & 1 & 1 & & 1 & & 2 & & & 1 & & & & & \\
\hline 39 & 4 & & & & & & & & & & & & & & & 1 & & 1 & & 1 & 1 & & & & & \\
\hline 40 & 2 & & & & & & & & & & & & & & & & & & & & & 1 & & 1 & & \\
\hline \multicolumn{27}{|l|}{41} \\
\hline \multicolumn{27}{|l|}{42} \\
\hline \multirow{2}{*}{\multicolumn{27}{|c|}{$\begin{array}{l}43 \\
44\end{array}$}} \\
\hline \multirow{2}{*}{\multicolumn{27}{|c|}{$\begin{array}{l}44 \\
45\end{array}$}} \\
\hline & & & & & & & & & & & & & & & & & & & & & & & & & & \\
\hline Total & 1277 & 16 & 42 & 67 & 57 & 64 & 42 & 34 & 49 & 61 & 82 & 106 & 124 & 111 & 102 & 83 & 63 & 55 & 45 & 31 & 18 & 10 & 4 & 7 & 3 & 1 \\
\hline
\end{tabular}


Aguas Profundas 2008, junto al Sr. Patricio Gálvez G. Jefe de proyecto en investigación de esta pesquería, tanto por la obtención de muestreo, información pesquera y la experiencia compartida en el estudio. Proyecto financiado por la Subsecretaría de Pesca de Chile. Por último se desea agradecer los comentarios y sugerencias de dos revisores anónimos.

\section{LITERATURA CITADA}

Abramov AA. 1990. Age and growth of two species of bigeyes, Epigonus angustifrons and E. elegans, from the Indian and Pacific Oceans. Voprosy Ikhtiologii 30(6): 1022-1028.

Andrews A \& D Tracey. 2007. Age validation of orange roughy and black cardinalfish using lead-radium dating. Final Report for Ministry of Fisheries Research Project DEE2005-02. Objective 1, 40 pp., National Institute of Water and Atmospheric Research, New Zealand.

Campana S. 2001. Accuracy, precision and quality control in age determination, including a review of the use and abuse of age validation methods. Journal of Fish Biology 59: 197-242.

CARE. 2006. Manual on generalized age determination. Procedures for groundfish. Committee of Age-Reading Experts Established 1982, 52 pp. Pacific Coast Groundfish Ageing Technicians. The Canada/U.S. Groundfish Committee. [en línea] <http://care.psmfc.org/docs/ CareManual2006.pdf>

Chilton D \& R Beamish. 1982. Age determination methods for fishes studied by the Groundfish Program at the Pacific Biological Station, Nanaimo. Special Publication, Canadian Journal of Fisheries and Aquatic Science 60: 1-102.

Cubillos LA, M Aguayo, M Neira, E Sanhueza \& C CastilloJordán. 2009a. Verificación de la edad y crecimiento de besugo Epigonus crassicaudus (de Buen, 1959) admitiendo error en la determinación de la edad. Revista de Biología Marina y Oceanografía 44(2): 417-427.

Cubillos LA, M Aguayo, C Castillo-Jordán, J Peñailillo, M Neira, E Sanhueza, M Pedraza-García \& M Salamanca. 2009b. Edad, crecimiento y mortalidad natural de besugo. Informe Técnico, Fondo de Investigación Pesquera FIPIT/2007-36: 1-150. [en línea] < http://www.fip.cl/FIP/ Archivos/pdf/informes/inffinal\%202007-36.pdf>

Gálvez M, H Rebolledo, C Pino, LA Cubillos, A Sepúlveda \& A Rojas. 2000. Parámetros biológico-pesqueros y evaluación de stock de besugo (Epigonus crassicaudus), 110 pp. Informe Final, Instituto de Investigación Pesquera, Talcahuano.

Gulland JA. 1971. The fish resources of the ocean, 255 pp. Fishing New Books, West Byfleet.
Hewitt DA \& JM Hoenig. 2005. Comparison of two approaches for estimating natural mortality based on longevity. Fishery Bulletin 103: 433-437.

Kozlowski J \& AT Teriokhin. 1999. Allocation of energy between growth and reproduction: the Pontryagin maximum principle solution for the case of age- and seasondependent mortality. Evolutionary Ecology Research 1: 423-441.

Leal E, F Contreras \& C Oyarzun. 2009. Distribución, batimetría y alimentación de Epigonus crassicaudus de Buen, 1959 (Perciformes: Epigonidae) en la costa de Chile. Gayana 73(1): 95-101.

Mitton JB \& WM Jr Lewis. 1989. Relationships between genetic variability and life-history features of bony fishes. Evolution 43: 1712-1723.

Neil H, P McMillan, D Tracey, R Sparks, P Marriott, C Francis \& L Paul. 2008. Maximum ages for black oreo (Pseudocyttus maculatus) and black cardinalfish (Epigonus telescopus) determined by the bomb chronometer method of radiocarbon ageing, and comments on the inferred life history of theses species. Final Research Report for Ministry of Fisheries research Project DEE2005/01: 1-63. National Institute of Water and Atmospheric Research, New Zealand.

Ojeda V \& M Aguayo. 1986. Edad y crecimiento de merluza del sur (Merluccius australis) (Gadiformes Meluccidae). Investigación Pesquera, Chile 33: 47-59.

Ojeda V \& L Muñoz. 2008. Técnicas y metodologías de análisis de edad en recursos pesqueros chilenos. Serie 1 peces de importancia comercial, 32 pp. Documento Técnico, Instituto de Fomento Pesquero, Valparaíso.

Parin NV. 1987. Oceanic ichthyocene systems and its commercial potential. En: NV Parin \& NP Novikov (eds). Biologicheskie resursy otkrytogo okeana (Biological resources of the open ocean), pp. 138-163. Nauka, Moscow.

Pfister CA \& FR Stevens. 2002. The genesis of size variability in plants and animals. Ecology 83(1): 59-72.

Prajneshu \& R Venugopalan. 1999. von Bertalanffy growth model in a random environment. Canadian Journal of Fisheries and Aquatic Sciences 56: 1026-1030.

Tracey DM, K George \& DJ Gilbert. 2000. Estimation of age and growth, and mortality parameters of black cardinalfish (Epigonus telescopus) in QMA 2 (East North Island). New Zealand Fisheries Assessment Report 2000/27: 1-21.

Wiff R, JC Quiroz, R Tascheri \& F Contreras. 2008. Effect of fishing tactics on the standardization of cardinalfish (Epigonus crassicaudus) catch rates in the demersal multispecies fishery off central Chile. Ciencias Marinas 34(2): 143-154. 